\title{
Precessional magnetization switching by a surface acoustic wave
}

\author{
L. Thevenard, ${ }^{1, *}$ I. S. Camara, ${ }^{1}$ S. Majrab,${ }^{1}$ M. Bernard, ${ }^{1}$ P. Rovillain, ${ }^{1}$ A. Lemaître,${ }^{2}$ C. Gourdon, ${ }^{1}$ and J.-Y. Duquesne ${ }^{1}$ \\ ${ }^{1}$ Sorbonne Universités, UPMC Universite de Paris 06, CNRS, Institut des Nanosciences de Paris, 4 place Jussieu, 75252 Paris, France \\ ${ }^{2}$ Laboratoire de Photonique et Nanostructures, CNRS, UPR 20, Route de Nozay, Marcoussis 91460, France
}

(Received 16 November 2015; revised manuscript received 3 March 2016; published 29 April 2016)

\begin{abstract}
Precessional switching allows subnanosecond and deterministic reversal of magnetic data bits. It relies on triggering a large-angle, highly nonlinear precession of magnetic moments around a bias field. Here we demonstrate that a surface acoustic wave (SAW) propagating on a magnetostrictive semiconducting material produces an efficient torque that induces precessional switching. This is evidenced by Kerr microscopy and acoustic behavior analysis in a $(\mathrm{Ga}, \mathrm{Mn})(\mathrm{As}, \mathrm{P})$ thin film. Using SAWs should therefore allow remote and wave control of individual magnetic bits at potentially $\mathrm{GHz}$ frequencies.
\end{abstract}

DOI: 10.1103/PhysRevB.93.134430

\section{INTRODUCTION}

The competition between solid-state devices and magnetic media for optimum data storage has driven the search for fast, energy-efficient, and cost-effective ways of addressing magnetization. Precessional magnetization switching is now widely accepted as a viable data-writing route, e.g., for spin valves in magnetic random access memory single data bits [1-4]. In precessional switching, a pulsed tickle field transverse to the initial magnetization triggers its precession around a bias field applied perpendicularly to the easy axis. The tickle field must be switched off at the right moment to obtain a final opposite magnetization. Switching can then be as short as half a precession period [5-9], potentially fractions of nanoseconds, but it will also occur for excitations lasting any of its odd multiples [20]. It is deterministic, and it requires lower fields than for classical Stoner-Wohlfarth coherent reversal [6]. The main challenge lies in the field-pulse generation. It is usually done inductively with a pulsed electrical or optical trigger [3,7-12]. Effective fields can also be realized by spin transfer torque. This exchange of angular momentum between a spin-polarized electrical current and the local magnetization [13-15] can be used to trigger the precessional switching of spin valves [4]. However, switching time distributions [16] and the necessity for large current densities remain serious challenges from both technological and fundamental points of view. To generate a pulsed effective field, another strategy consists in using the coupling between magnetization and strain in a magnetoelastic material. Here we demonstrate experimentally on an out-of-plane magnetized layer an original approach: a $548 \mathrm{MHz}$ surface acoustic wave (SAW) induces efficient precessional switching. This is an exciting perspective since the wave properties of SAWs open up the possibility to rely on focusing or interferences to switch magnetization selectively.

This proof of concept was done on a dilute magnetic semiconductor, $(\mathrm{Ga}, \mathrm{Mn})(\mathrm{As}, \mathrm{P})$, in which the ferromagnetic phase is carrier-mediated, thus enabling magnetoelasticity [17]. In this material, picosecond acoustic pulses [18] and surface acoustic waves [19] can trigger or force a small amplitude precession of the magnetization when it is resonantly

\footnotetext{
*thevenard@insp.jussieu.fr
}

coupled to the strain. For large enough strain, the precession could reach very large amplitudes, and eventually lead to the full reversal of magnetization [20]. For this, using SAWs presents several advantages. They are particularly well suited to the magnetostriction of $(\mathrm{Ga}, \mathrm{Mn})(\mathrm{As}, \mathrm{P})$, since a Rayleigh wave propagating along $\langle 110\rangle$ exhibits strain components that couple directly to the uniaxial out-of-plane and in-plane anisotropies [19,20]. SAWs can be made quasimonochromatic, yielding the high-power spectral density required for this intrinsically resonant mechanism, instead of the large bandwidth excitation of a current pulse. SAWs can be generated electrically using interdigitated transducers, a well-mastered technology, being routinely used as bandpass filters in the gigahertz range in modern electronics. They can reduce coercivity in planar $\mathrm{FeGa}$ [21] and in $(\mathrm{Ga}, \mathrm{Mn})(\mathrm{As}, \mathrm{P})$ [22] by assisting the nucleation/propagation of domains. This thermally activated process involving a poorly controlled waiting time [23] could be advantageously replaced by a deterministic mechanism relying on precessional switching, in view of possible strain-based magnetostrictive memories.

\section{EXPERIMENTAL METHODS}

The sample is a 50-nm-thick layer of $(\mathrm{Ga}, \mathrm{Mn})(\mathrm{As}, \mathrm{P})$ chosen for its low out-of-plane anisotropy (yielding low magnetic precession frequencies [19]), yet square hysteresis loops. Its Curie temperature is $95 \mathrm{~K}$. A piezoelectric layer was sputtered onto the magnetic layer and interdigitated transducers were lithographed, yielding an acoustic wavelength of $5 \mu \mathrm{m}$ corresponding to a frequency $f_{\mathrm{SAW}}=548 \mathrm{MHz}$ [Fig. 1(a)]. Set $2 \mathrm{~mm}$ apart, they excite and detect the acoustic waves. The maximum excited strain is of the order of $5 \times 10^{-4}$, estimated using a vector network analyzer (VNA). To observe magnetoacoustic switching, the SAW excitation setup is coupled to a polar Kerr microscope, sensitive to the out-of-plane component of the magnetization. More details on the sample and setup are given in Appendix.

An out-of-plane permanent magnet is used to initialize the magnetization ("up" or "down") of the sample. The static bias field is then very carefully aligned in the plane of the sample to avoid any spurious domain-wall nucleation/propagation. Bursts of acoustic waves are generated thanks to a pulsed radiofrequency (rf) excitation (carrier frequency $548 \mathrm{MHz}$ ). The setup allows two operating modes: (i) single-shot SAW 

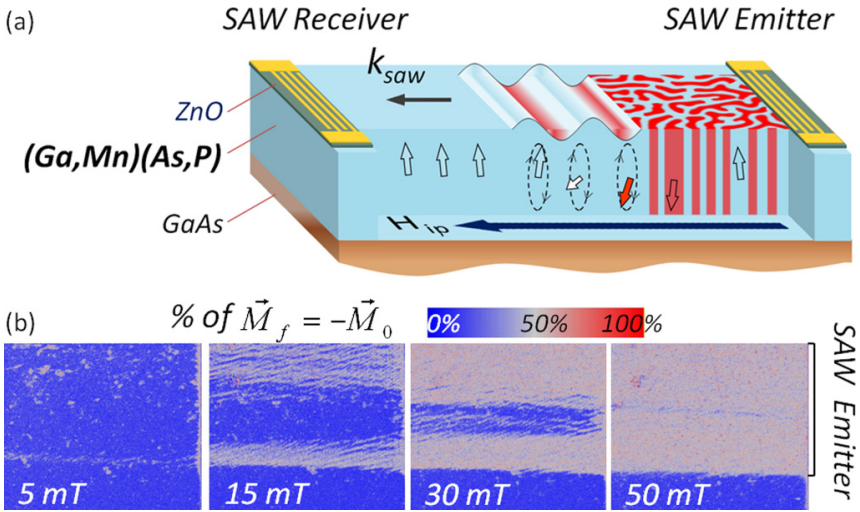

FIG. 1. (a). Schematics (not to scale): The magnetization is initialized "up" $\left(\vec{M}_{0}\right)$. An in-plane field $H_{\text {ip }}$ tilts it toward the plane and brings the Mn spin precession frequency into resonance with the SAW, thus triggering magnetization precession. (b) Kerr images $\left(268 \times 360 \mu \mathrm{m}^{2}\right)$ after a single 700-ns-long radiofrequency $(\mathrm{rf})$ power burst applied under various in-plane fields. The incident power is $P_{0}=8.9 \mathrm{~W}$ and $T=20 \mathrm{~K}$. After normalization to fully saturated "up" and "down" states, the color coding indicates the percentage of final magnetization $\left(\vec{M}_{f}\right)$ that lies opposite the initial one.

burst, (ii) repetitive bursts (repetition frequency $1 \mathrm{kHz}$ ). We first present experiments done in mode (i).

\section{RESULTS}

\section{A. Single acoustic pulse switching}

The temperature is set to $T=20 \mathrm{~K}$ and the sample is initialized "down." A single SAW burst is emitted under a constant in-plane magnetic field $\mu_{0} H_{\text {ip }}$ [Fig. 1(a)]. The incident $\mathrm{rf}$ burst has a power of $P_{0}=8.9 \mathrm{~W}$ and a duration of $\tau=700 \mathrm{~ns}$. After the field has been brought back to zero, we take a Kerr image and normalize it to "up" and "down" saturation images.

For each pixel, a normalized intensity is calculated as $\Delta m_{z}=\frac{I_{\mathrm{SAW}}-I_{\mathrm{down}}}{I_{\mathrm{up}}-I_{\mathrm{down}}}$. This is equal to the local magnetization change $\Delta m_{z}$ (convoluted with our $\approx 1 \mu \mathrm{m}$ optical resolution [24]), which we color-code between blue and red (0-100\%). A local $\Delta m_{z}=0 \%$ (coded blue) signifies that the magnetization has remained "down"; $\Delta m_{z}=100 \%$ (coded red) signifies that it has switched "up." $\Delta m_{z}=50 \%$ (coded white) means that there are an equal number of unresolved up and down magnetized domains. Indeed, the layer's out-of-plane easy axis rules out the possibility of the magnetization being in-plane in zero field.

The effect of the SAW on the magnetization can be directly seen in Fig. 1(b) for different in-plane fields $\mu_{0} H_{\text {ip }}$ applied during the SAW burst. A switching phenomenon clearly takes place on part of the image. At $30 \mathrm{mT}$, almost the whole area on the SAW path has been affected, except for a central region, located in front of a large defect on the emitting interdigitated transducer (not visible on the image), and where the SAW amplitude is presumably much lower. Switching initiates at very low field $(5 \mathrm{mT})$ close to the emitter (where the fabrication procedure has made the layer softer) and at the edge of the SAW wavefront, where the surface displacement is higher due to the finite aperture of the transducer [25]. At $\mu_{0} H_{\mathrm{ip}}=50 \mathrm{mT}$,
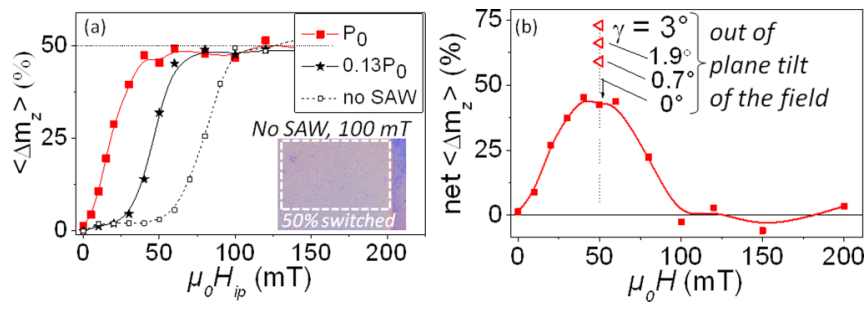

FIG. 2. (a) Relative magnetization changes $\left\langle\Delta m_{z}\right\rangle$ averaged on the dashed white rectangle, without SAW (empty squares) or with SAW. Inset: normalized Kerr image of the control experiment without any SAW [same color-coding as in Fig. 1(b)]. (b) Net effect of the SAW at $P_{0}$, i.e., the difference between full and empty squares of (a). Empty triangles give the net effect of an out-of-plane tilt of the field [50 mT, corresponding images in Fig. 5(a)].

switching occurs on the entire SAW path. These observations are identical when the sign of the initial saturation and/or of $\mu_{0} H_{\text {ip }}$ is inverted. No effect is observed at all when the interdigitated transducer is excited $20 \mathrm{MHz}$ off resonance (no SAW generated, but inductive emission of an rf field). This rules out the influence of radiated electromagnetic fields, as well as the Joule effect and Foucault losses in the metallic bodies. Finally, at $\mu_{0} H_{\mathrm{ip}}=50 \mathrm{mT}$, switching is observed over the $2 \mathrm{~mm}$ separating excitation/detection transducers for $\mathrm{rf}$ excitation bursts down to $\tau=100 \mathrm{~ns}$ long. This is shorter than the acoustic burst rise time, so that for $\tau<100 \mathrm{~ns}$ the maximum strain does not reach its switching threshold value, and reversal is only observed at the edge of the wavefront where the SAW amplitude is the greatest. Similar experiments were run for decreasing rf powers.

At this point, we should emphasize that the white color in the normalized images of Fig. 1(b) indicates that, on average, $50 \%$ of the spins have switched orientation. This is quantified in Fig. 2(a), where $\Delta m_{z}$ is averaged on the SAW path and plotted as a function of $\mu_{0} H_{\mathrm{ip}}$, and for two different rf powers. $\left\langle\Delta m_{z}\right\rangle$ converges to $50 \%$ at around $50 \mathrm{mT}$ for $P_{0}(80 \mathrm{mT}$ for $0.13 P_{0}$ ). This may appear counterintuitive since one would expect either no reversal or complete reversal in a precessional switching picture. We first consider a possible temperature rise during SAW propagation, since part of the acoustic loss results in incoherent phonons production (thermoelastic or Akhieser effects). A crude estimation shows this effect is in fact responsible for a maximum temperature rise of $0.02 \mathrm{~K}$ (see details in Appendix B). Another option would be a transient lowering of the anisotropy by the SAW, sufficient to bring the magnetization fully in-plane, from where it could return pointing either "up" or "down." Using $20 \mathrm{~K}$ parameters, however, we calculate that under $50 \mathrm{mT}$ and for a SAW amplitude of $5 \times 10^{-4}$, the magnetization is still at $55.7^{\circ}$ from the sample normal, ruling out this possibility. Instead, these results are the signature of SAW-induced precessional switching, of which we briefly recall the mechanism.

\section{B. Principles of precessional switching}

In a macrospin picture, the interaction between magnetization and a surface acoustic wave leads to a small-angle precession forced by the rf field generated through inverse 


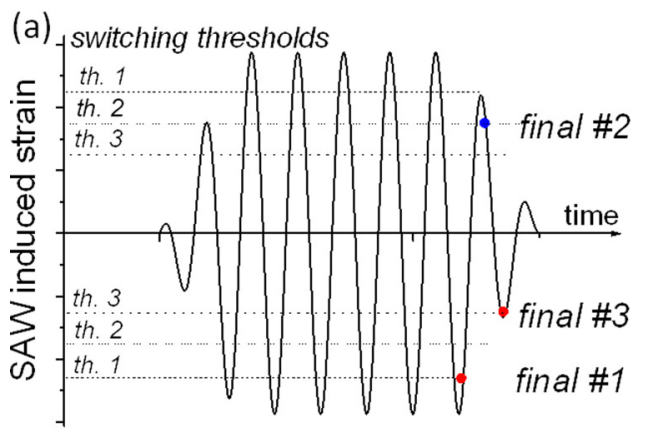

(b)

Initialization
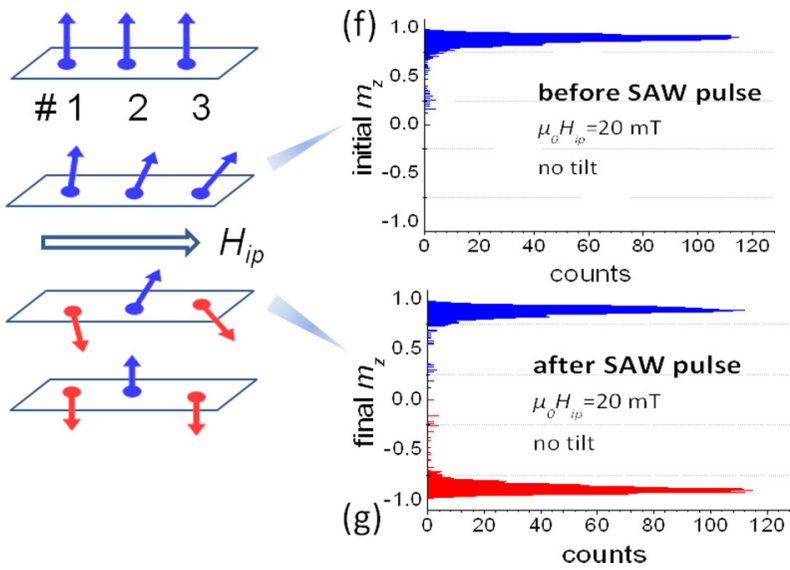

FIG. 3. (a)-(e) Schematics of a SAW strain pulse and associated macrospin behavior leading to SAW-assisted precessional switching in the presence of anisotropy dispersion. The bias field $\mu_{0} H_{\text {ip }}$ lies in the plane of the sample. The magnetization dynamics goes from precessing around its equilibrium position to precessing around the applied field $\left(\mu_{0} H_{\mathrm{ip}}\right)$ when the strain crosses a particular threshold (dashed lines). At the end of the SAW pulse, the final magnetization configuration is dictated by the sign of the strain upon last crossing the threshold (red/blue dots). Macrospin simulations (under $20 \mathrm{mT}$ ): distribution of initial (f) and final (g). Both histograms cumulate 11659 counts.

magnetostriction by the acoustic pulse [20]. This effective field is expressed as $h_{\mathrm{SAW}}^{\mathrm{rf}}(t)=\sin 2 \theta_{0} f\left(\varphi_{0}, A_{2 \varepsilon}, A_{2 x y}, \varepsilon_{x x}(t), \varepsilon_{z z}(t)\right)$, where $\theta_{0}, \varphi_{0}$ are the equilibrium magnetization polar and azimuthal angles and $f$ is a function that depends on the magnetoelastic coefficients $A_{2 \varepsilon}, A_{2 x y}$ and the SAW-induced strains $\varepsilon_{x x}(t), \varepsilon_{z z}(t)$ (see Appendix $C$ for the explicit expression). $\vec{h}_{\mathrm{SAW}}^{\mathrm{rf}}(t)$ is at all times in the plane normal to the magnetization, which is convenient as it then maximizes the resulting torque. A static in-plane field $\mu_{0} H_{\mathrm{ip}}$ is necessary to both pull the magnetization toward the plane (so that $\sin 2 \theta_{0} \neq 0$ ) and to adjust the Mn precession frequency to $f_{\text {SAW }}$. At this particular field, a resonance has been observed in the acoustic velocity and amplitude variations [19]. This SAW-induced ferromagnetic resonance (SAW-FMR) regime has also been seen in nickel [26,27]. In all these experiments, the finite damping of the materials, as well as the small dynamic strain amplitudes, inherently limited the precession amplitude at resonance.

A second regime of this magnetoacoustic interaction exists, however [20], as depicted in Fig. 3. For a SAW amplitude and/or a static field larger than a particular threshold, the magnetization then precesses around the applied field, rather than around $\theta_{0}$ [Fig. 1(a)]. This is the onset of precessional switching [6]. The threshold depends on the SAW amplitude and frequency detuning with respect to the precession frequency, itself governed by the applied field and the anisotropy constants, in particular by the dominant magnetoelastic term $A_{2 \varepsilon}$. At the end of the SAW burst, the generated strain gradually decreases, following the interdigitated transducer transient, and the amplitude of the magnetization precession decreases accordingly. When the SAW amplitude drops below the threshold, the magnetization starts precessing around its final position ("up" or "down"), which depends on the SAW phase with respect to the precession dynamics, very much akin to the influence of the trailing field pulse edge in traditional precessional switching experiments $[3,8,10,11]$. If the sample were an ideal collection of noninteracting macrospins with a unique precession frequency, seeing the same (but time- delayed) acoustic burst, the final state would be $0 \%$ or $100 \%$ switched. However, demagnetizing effects and micromagnetic parameter dispersion are well known to induce nonuniform precessional switching [28,29], as depicted schematically in Fig. 3 and discussed below.

\section{Effect of anisotropy dispersion}

We estimated experimentally this dispersion, in particular that of the magneto-elastic coefficient $A_{2 \varepsilon}$ responsible for the out-of-plane anisotropy. This was done by analyzing spatially the saturation field of a first magnetization curve taken without SAW: after "up" or "down" saturation, the in-plane field $\mu_{0} H_{\mathrm{ip}}$ is ramped up and Kerr images acquired at each field. Instead of averaging the Kerr intensity $I$ over the large area in front of the IDT, it is averaged over $10 \times 10$ pix $^{2}\left(2.6 \times 2.6 \mu \mathrm{m}^{2}\right)$ bins on the image. These $I\left(\mu_{0} H_{\mathrm{ip}}\right)$ curves are then analyzed numerically to extract a local saturation field, i.e., the field required to align the magnetization in the plane of the sample. Figure 4(a) shows a color map of these saturation fields. They are distributed rather randomly in the image, and the resulting histogram [Fig. 4(b)] shows a flattened Gaussian distribution, centered around $\left\langle\mu_{0} H_{\text {sat }}\right\rangle=111$ and $26 \mathrm{mT}$ wide. This wide dispersion was probably caused by the deposition of
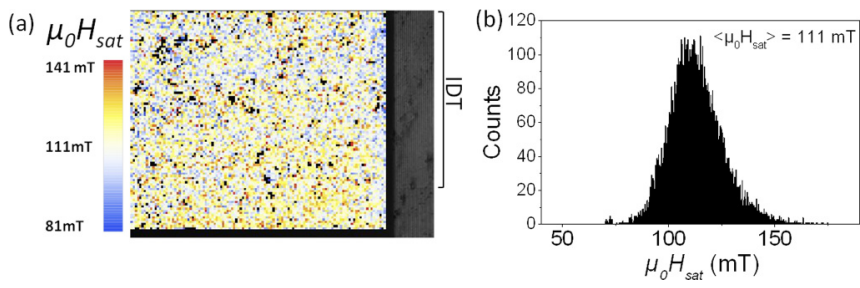

FIG. 4. (a) Color map of the in-plane saturation field distribution $(T=20 \mathrm{~K})$, in $2.6 \times 2.6 \mu \mathrm{m}^{2}$ bins. Black bins indicate that the data were locally too poor to extract a saturation field. (b) Histogram of the saturation fields. 
the piezoelectric layer. We had already shown it to deteriorate the Curie temperature [19,38].

The magnetization at saturation being 3.5 smaller than the dominant out-of-plane magnetic anisotropy field, the dispersion in saturation fields mainly reflects that of the out-of-plane anisotropy and not of the shape anisotropy. In turn, this will affect the dispersion of the initial magnetization position, of the precession frequency, of the detuning to the SAW frequency, and hence of the final state after the SAW burst, as described schematically in Figs. 3(a)-3(e). To test whether this dispersion could be responsible for the final relative "up"/“"down" spin proportion, we simulated the effect of a single SAW burst, following the macrospin methodology developed in Ref. [20]. An illustration at $20 \mathrm{mT}$ is shown in Figs. 3(f) and 3(g). Feeding the experimental anisotropy dispersion of Fig. 4(b) and $T=20 \mathrm{~K}$ micromagnetic parameters, we first calculate the distribution of the initial magnetization position [Fig. 3(f)]. Assuming a 700-ns-long burst SAW pulse of amplitude $\varepsilon_{\max }=5 \times 10^{-4}$, we then calculated numerically the distribution of final states under field, and we found it to be indeed an ensemble of "up" and "down" spins in equal numbers [Fig. 3(g)]. Although this simulation is a macrospin limit of the experiment and as such does not account for demagnetizing and exchange effects, it gives an excellent qualitative account of what occurs: at the end of the SAW burst, the dispersion in switching thresholds leads to equiprobable "up" or "down" end positions - and the formation of numerous domains giving $50 \%$ magnetization changes on average.

\section{Net effect of the SAW}

Finally, we estimate the net effect of the SAW by comparing the previous measurements to data taken under an in-plane field alone, with no SAW burst sent. Toward that end, a set of control images is taken, where the field $\mu_{0} H_{\text {ip }}$ is ramped up and brought back to zero without any SAW [open symbols in Fig. 2(a)]. In this situation, the magnetization follows the effective field (applied field competing with the anisotropy field). For low fields (below $50 \mathrm{mT}$ ), the magnetization remains almost fully "up." However, for large fields (above $100 \mathrm{mT}$ ), the magnetization has been pulled into the plane. As the applied field is brought back to zero, "up" and "down" domains have equal probability to form, giving a final average $\left\langle m_{z}\right\rangle \approx 0$, i.e., $\left\langle\Delta m_{z}\right\rangle=50 \%$. This is encouraged by the fact that an out-of-plane magnetized layer will tend to self-organize into "up"/“down" domains. In our sample, the self-organization period has been estimated to be $690 \mathrm{~nm}$ at $20 \mathrm{~K}$ [30]. The domains are therefore smaller than our $\approx 1 \mu \mathrm{m}$ resolution, which appears as white pixels [inset of Fig. 2(a)]. The gradual slope of the curve without SAW in Fig. 2(a) reflects the large dispersion of saturation fields. The net effect of the SAW can then be calculated as the difference between the percentage of area switched $\left\langle\Delta m_{z}\right\rangle$ with and without SAW [Fig. 2(b)]. It exhibits a broad maximum at $50 \mathrm{mT}$ for $P_{0}$ (at $0.13 P_{0}$, it peaks at $60 \mathrm{mT}$ ). At high fields, the final state is the same with and without SAW, an average zero magnetization attributed to a collection of "up" and "down" sub- $\mu$ m domains. This multidomain interpretation is confirmed by tilting the field out of the plane by an angle $\gamma=0.7^{\circ}, 1.9^{\circ}$, and $3^{\circ}$. This creates a slight imbalance between up and down spin populations
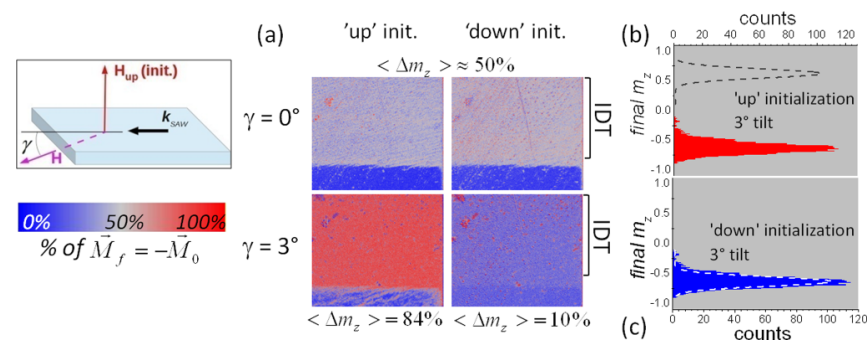

FIG. 5. (a) Normalized Kerr images $\left(268 \times 360 \mu \mathrm{m}^{2}\right)$ after a single SAW pulse $P_{0}$ at $T=20 \mathrm{~K}$ under $\mu_{0} H=50 \mathrm{mT}$, with or without tilt and for "up" or "down" initialization with the corresponding reversal percentages averaged on the SAW path. $\vec{M}_{0}$ $\left(\vec{M}_{f}\right)$ are the initial (final) local magnetizations. (b) Macrospin simulations of the final state after a SAW burst, for two different initialization distributions (dashed lines). The tilt induces $100 \%$ switching when it points opposite the initialization direction.

[Fig. 5(a)]. The empty triangular symbols in Fig. 2(b) show that for an incident SAW power $P_{0}$ and a field of $50 \mathrm{mT}$, the net switched magnetization can then reach up to $73 \%$ for a tilt of $\gamma=3^{\circ}$.

To confirm that these results are in keeping with the precessional switching mechanism, we performed as above macrospin simulations of the final magnetization state. The experimental out-of-plane anisotropy dispersion evidenced in Fig. 4(b) and experimental conditions of Fig. 5(a) were used: $\mu_{0} H=50 \mathrm{mT}$ and a $3^{\circ}$ out-of-plane tilt. This time, the final-state distribution is not $50 \%$ "up"/“down" as in the calculation run without tilt, but it is now completely shifted to the direction of the minute out-of-plane component of the static field [Fig. 5(b)], giving either $100 \%$ or $0 \%$ switching. Given the approximations of this calculation, it is not surprising that it does not fully match the experimentally observed $84 \%$ and $10 \%$ switching [Fig. 5(a)], but it nevertheless once more gives an excellent description of what is happening in the layer.

\section{E. Acoustic and magnetic nonlinearities}

We now demonstrate experimentally that the process responsible for the maximum net $50 \%$ switched state (for $\gamma=0^{\circ}$ ) results from SAW-induced precessional switching, represented schematically in Fig. 1(a). A traditional test of this mechanism is the influence of the pulse duration on the final magnetization state [28,31]. The precession frequency dispersion makes this test irrelevant in our sample. We therefore searched for another decisive signature, namely the appearance of nonlinearities in the acoustics dynamics. They can be tracked by measuring the acoustic pulse amplitude and phase at the receiving transducer [Fig. 1(a)] as a function of applied field. These measurements are done in repetitive mode, with about 2000 signal averages required for each field data point. The pulse modulation enables a clear separation in the time domain of the main acoustic signal and the crosstalk signal (electromagnetic wave from the emitting transducer). At low incident powers, the attenuation variations $\Delta \Gamma$ versus applied field vary weakly with power - the signature of linear behavior [e.g., the $1.4 \times 10^{-5} P_{0}$ curve shown in Fig. 6(a)]. A clear resonance at $85 \mathrm{mT}$ is observed, precisely the field calculated to yield a precession frequency equal to 

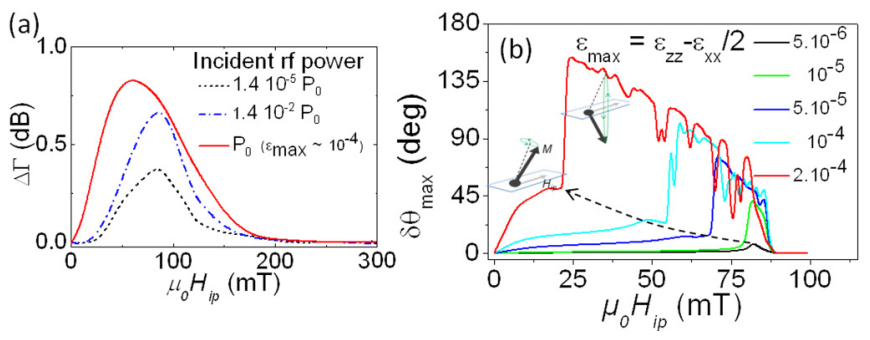

FIG. 6. Acoustic and magnetic nonlinearities $(T=20 \mathrm{~K})$. (a) SAW FMR data: variation of acoustic attenuation $\Delta \Gamma$ measured in transmission by the receiving interdigitated transducer. (b) Numerical calculations: angular out-of-plane precession amplitude obtained from the numerical solution to the LLG equation in the presence of SAW. Precessional switching starts at the slope kink and occurs at lower fields for increasing strain.

$f_{\text {SAW }}$, as observed in Ref. [19]. This is a frequency-domain signature of SAW-induced precession. For medium powers (e.g., $1.4 \times 10^{-2} P_{0}$ ), the attenuation at resonance increases with power, signifying an increase of the fraction of energy being transferred to the magnetic system. Concomitantly, the acoustic-wave velocity exhibits a similar behavior (not shown). It is also at these powers that a significant magnetization reversal starts to be observed in single-shot mode [32]. At high powers (e.g., $P_{0}$ ), the resonance widens and shifts toward lower fields. This downshift and distortion of the resonance are well-known features of large-angle precession observed in high-power cavity FMR, e.g., in garnet films [33-35]. In this nonlinear regime, the small-angle solution to the LandauLifshitz-Gilbert (LLG) equation is not valid anymore. The large precession modifies the demagnetization contribution to the total energy, which in turn modifies the precession frequency. These observations hint at strongly nonlinear-and possibly chaotic-magnetization dynamics.

To confirm this, the angular amplitude of the precession, $\delta \theta_{\max }=\operatorname{Max}\left[\theta(t)-\theta_{0}\right]$, was therefore calculated as a function of in-plane field for various SAW amplitudes [Fig. 6(b)], using the macrospin approach [20]. The onset of precessional switching is characterized by a kink in the $\delta \theta_{\max }\left(\mu_{0} H_{\mathrm{ip}}\right)$ slope, and it occurs at lower fields for increasing strain, as observed experimentally. For a large dynamic strain $\left(2 \times 10^{-4}\right)$, the numerical calculation shows that macrospin switching occurs as early as $20 \mathrm{mT}$, close to our experimental observations [ $P_{0}$ curve in Fig. 2(a)]. It is interesting to compare these calculations to the cavity FMR experiments taken on yttrium iron garnet (YIG) samples by Gnatzig [33] et al. Their measurement of precession amplitude versus applied field up to high rf powers is strikingly similar to the magnetization dynamics we calculate up to large SAW amplitudes [Fig. 6(b)]. These similarities are conclusive of the concomitant acoustic and magnetic nonlinearities when magnetization reversal is observed, and they open up the possibility of SAW-induced magnetization switching in YIG, a technologically relevant weakly damped ferromagnet.

\section{F. Tuning the switching efficiency with the in-plane field direction}

A last illustration of the link between SAW attenuation and magnetization switching efficiency is done by rotating the field in-plane with respect to the SAW wave vector by an angle $\beta$. Because $(\mathrm{Ga}, \mathrm{Mn})(\mathrm{As}, \mathrm{P})$ presents an in-plane anisotropy of magnetoelastic origin [36,37], the acoustic attenuation should vary with $\beta$ (see the theory in Appendix C). SAW-FMR experiments done in repetitive mode show that the SAW attenuation at resonance does decrease continuously with increasing $\beta$ [Fig. 7(a)]. We then performed single acoustic pulse switching experiments at $\mu_{0} H_{\mathrm{ip}}=30 \mathrm{mT}$ and $P=$ $0.48 P_{0}$, varying $\beta$. The normalized Kerr images [Fig. 7(b)] clearly show a monotonously decreasing switching efficiency as the field is turned away from the acoustic wave-vector direction, in line with the corresponding decrease in the SAW FMR amplitude at resonance [Fig. 7(c)].

\section{CONCLUSION}

To summarize, we have observed SAW-induced precessional switching on out-of-plane magnetized layers of $(\mathrm{Ga}, \mathrm{Mn})(\mathrm{As}, \mathrm{P})$. Switching was observed for $\mathrm{rf}$ pulses down to $100 \mathrm{~ns}$, but in theory it could occur much closer to the fundamental half-precession period limit by optimizing the interdigitated transducer's transient. Reversal efficiencies above $50 \%$ could be obtained on samples showing weakly dispersed magnetic anisotropy, or samples that have been micro- or nanopatterned. Finally, this mechanism could also be used

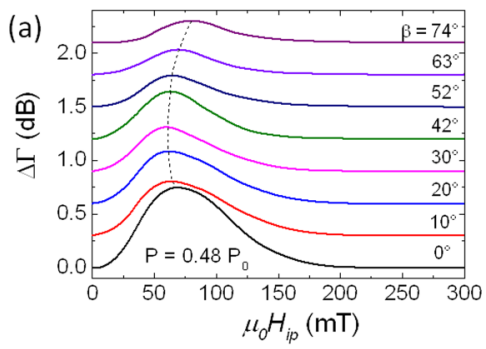

(b)

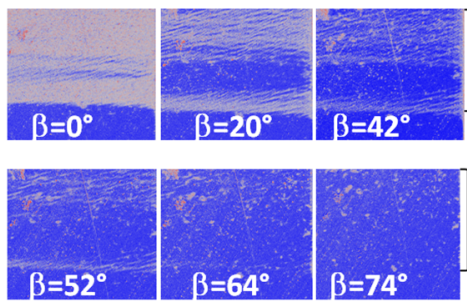

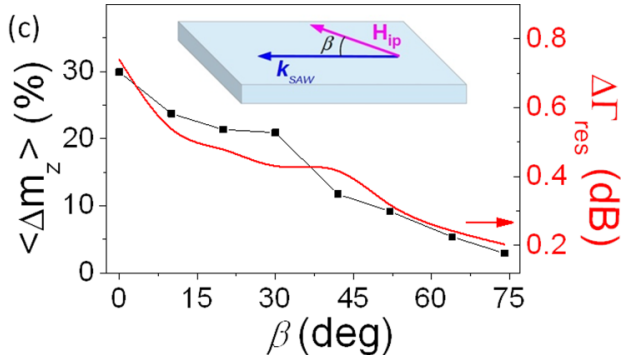

FIG. 7. Effect of rotating the in-plane field with respect to $\vec{k}_{\mathrm{SAW}}\left(T=20 \mathrm{~K}, P=0.48 P_{0}\right)$. (a) SAW FMR as a function of the angle $\beta$ between the SAW wave vector and the field, measured in repetitive mode. Curves shifted vertically for clarity. (b) Normalized Kerr images [268 $\times 360 \mu \mathrm{m}^{2}$, same color-coding as in Fig. 1(b)]. Brackets indicate the position of the SAW emitter. (c). Field-angle dependence of the switching efficiency averaged on the SAW path (black squares, left $y$ axis, $\mu_{0} H_{\mathrm{ip}}=30 \mathrm{mT}$ ), and of the acoustic attenuation changes at resonance $\Delta \Gamma_{\text {res }}$ (red line, right $y$-axis), from curves in (a). 
on in-plane magnetized samples, provided their precession frequency can be tuned to an experimentally accessible SAW frequency.

\section{ACKNOWLEDGMENTS}

We thank L. Becerra for the lithography of the sample, J. von Bardeleben for the cavity FMR data, J.-Y. Prieur for insightful advice, and B. Jusserand and T. Cren for their critical reading of the manuscript. This work has been supported by the French Agence Nationale de la Recherche (ANR13-JS040001-01).

\section{APPENDIX}

\section{Sample and setup details}

The layer was grown by molecular beam epitaxy. The polar Kerr microscopy was done at $600 \mathrm{~nm}$ with an objective of 0.4 numerical aperture. The field $\mu_{0} H_{\text {ip }}$ was applied in the plane of the sample with an air-cooled CAYLAR rotating dipole. The piezoelectric layer consists of $250 \mathrm{~nm}$ of $\mathrm{ZnO}$, deposited over $70 \mathrm{~nm}$ of $\mathrm{SiO}_{2}$, necessary for good adhesion. The thickness of the $\mathrm{SiO}_{2}$ layer was designed to increase the Kerr contrast [38]. The interdigitated transducers (IDTs) are made out of $\mathrm{Cr} / \mathrm{Au}$ (thickness $10 / 80 \mathrm{~nm}$ ) with a metallization ratio of 0.5 and teeth width $1.25 \mu \mathrm{m}$. The large number of teeth (60 pairs) gives a reasonably narrow resonance width of $8 \mathrm{MHz}$, but a long transient of about $400 \mathrm{~ns}$. Finally, a window was etched in the $\mathrm{ZnO}$ layer between the two IDTs in order to do the Kerr imaging.

\section{Estimation of SAW-induced temperature rise}

Here we establish an upper boundary for the temperature rise associated with the passage of the acoustic wave at 30 $\mathrm{K}$. The incident rf power is $P_{0}=8.9 \mathrm{~W}$, which is converted into acoustic power $P_{\mathrm{ac}}=\eta P_{0}$ with the electromechanical conversion factor $\eta=0.012$ estimated by VNA. The energy of the emitted $\tau=700 \mathrm{~ns}$ SAW burst is $E_{0}=\tau P_{\text {ac }}$. After propagating a distance $l$ from the IDT, it has decreased to $E=\alpha E_{0}$, where $\alpha$ is given by $-\frac{10}{l} \log (\alpha)=\Gamma$. We take $\Gamma=14 \mathrm{~dB} \mathrm{~cm}^{-1}$ from Slobodnik et al. [39]. Note that this value was measured at $300 \mathrm{~K}$ and $1 \mathrm{GHz}$ and is therefore a very conservative upper boundary for our low-temperature, $548 \mathrm{MHz}$ experiments. The dissipated energy is $Q=E_{0}-E=(1-\alpha) E_{0}$. The volume swept by the SAW is $v=l w \lambda_{\mathrm{SAW}}$, where $w=1 \mathrm{~mm}$ is the IDT aperture. The resulting calorific capacity is $C=\rho c v$, where $c=40 \mathrm{~J} \mathrm{~kg}^{-1} \mathrm{~K}^{-1}$ is the specific heat [40] at $30 \mathrm{~K}$.

If we suppose the entire dissipated acoustic energy is transformed into the volume $v$ and that this heat is not evacuated at all, the mean temperature rise then reads $\Delta T=$ $\frac{Q}{C} \approx 0.02 \mathrm{~K}$ for $l$ between $1 \mu \mathrm{m}$ and $2 \mathrm{~mm}$. This is a crude model giving an upper boundary of the temperature rise. It is negligible for the magnetization reversal physics we are considering.

\section{Influence of in-plane anisotropy on SAW FMR}

In Ref. [19], the SAW-FMR theory was developed for $(\mathrm{Ga}, \mathrm{Mn})(\mathrm{As}, \mathrm{P})$ neglecting the effect of the SAW on the uniaxial in-plane anisotropy, $A_{2 x y}$. Here we include $A_{2 x y}$ to explain why the attenuation variations are expected to change when the angle of the field with the SAW wave vector is varied [Figs. 7(a) and 7(c)]. The total energy can be expressed as $E_{\mathrm{tot}}=W+M_{s} F_{\text {stat }}+M_{s} F_{\mathrm{ms}, \mathrm{SAW}}$, with the different contributions: the purely elastic term $W=\frac{1}{2} c_{i j k l} \varepsilon_{i j} \varepsilon_{k l}$, the term governing the static magnetization configuration $F_{\text {stat }}$, and the SAW-induced magnetostrictive term $F_{\mathrm{ms}, \mathrm{SAW}}$ :

$$
\begin{aligned}
F_{\text {stat }}= & -\mu_{0} H_{\mathrm{ip}}\left[\left(m_{x} \cos \beta+m_{y} \sin \beta\right) \sin \theta\right] \\
+ & {\left[B_{d}-3 B_{c}+\Delta \varepsilon_{0}\left(A_{2 \varepsilon}+A_{4 \varepsilon}\right)\right] m_{z}^{2} } \\
+ & {\left[\frac{5}{2} B_{c}+\frac{1}{2} A_{4 \varepsilon} \Delta \varepsilon_{0}\right] m_{z}^{4}-\left(m_{x}^{4}+m_{y}^{4}\right)\left[B_{c}-A_{4 \varepsilon} \Delta \varepsilon_{0}\right] } \\
+ & \frac{1}{2} A_{2 x y} \varepsilon_{X Y, 0}\left(m_{x}^{2}-m_{y}^{2}\right), \\
F_{\mathrm{ms}, \mathrm{SAW}}= & \Delta \varepsilon(t)\left(A_{2 \varepsilon}+A_{4 \varepsilon}\right) m_{z}^{2}+\frac{1}{2} A_{4 \varepsilon} \Delta \varepsilon(t) m_{z}^{4} \\
& +A_{4 \varepsilon} \Delta \varepsilon(t)\left(m_{x}^{4}+m_{y}^{4}\right)-\frac{1}{4} A_{2 x y} \varepsilon_{x x}(t)\left(m_{x}^{2}-m_{y}^{2}\right) .
\end{aligned}
$$

The components of the unit magnetization vector are defined as $m_{i}=M_{i} / M_{s}(i=x, y, z)$, with $\vec{x} / / \vec{k}_{\mathrm{SAW}} . H_{\mathrm{ip}}$ is the in-plane field applied at an angle $\beta$ from [1-10]. $B_{d}=\frac{\mu_{0} M_{s}}{2}$ is the demagnetizing field and $B_{c}$ is the cubic anisotropy constant. The magnetostrictive coefficients are $A_{2 \varepsilon}, A_{4 \varepsilon}$ (firstand second-order uniaxial out-of-plane anisotropy), and $A_{2 x y}$ (uniaxial in-plane anisotropy distinguishing in-plane [110] and [1-10] axes). The strain felt by the layer has both a static component due to the pseudomorphic deposition of the magnetic layer on the GaAs substrate $\left(\varepsilon_{X X, 0}=\varepsilon_{Y Y, 0}\right.$ and $\varepsilon_{Z Z, 0}$, with $\Delta \varepsilon_{0}=\varepsilon_{Z Z, 0}-\varepsilon_{X X, 0}$ expressed in the $(X, Y, Z)$ reference frame with $X / /[100])$, and a dynamic SAW-induced strain $\left(\varepsilon_{x x}(t), \varepsilon_{z z}(t)\right.$, with $\left.\Delta \varepsilon(t)=\varepsilon_{z z}(t)-\frac{\varepsilon_{x x}(t)}{2}\right)$. Finally, $c_{i j k l}$ are the components of the elastic constant tensor, and $\varepsilon_{i j}$ are those of the strain.

As in Ref. [19] and following Dreher et al. [41], we define a second reference frame $(1,2,3)$ where $\vec{m}_{3}$ is aligned with the static magnetization [polar coordinates $\left(\theta_{0}, \varphi_{0}\right)$ ]. The dynamic rf field generated by inverse magnetostriction is defined by $\mu_{0} h_{i}=-\left.\frac{\partial F_{\mathrm{ms}, \mathrm{SAW}}}{\partial m_{i}}\right|_{\vec{m}=\vec{m}_{3}}$ and lies in the plane perpendicular to the static magnetization:

$$
\begin{gathered}
\mu_{0} h_{1}(t)=\Delta \varepsilon(t) \sin 2 \theta_{0}\left[\left(A_{2 \varepsilon}+A_{4 \varepsilon}\right)+A_{4 \varepsilon} \cos ^{2} \theta_{0}\right] \\
-A_{4 \varepsilon} \Delta \varepsilon(t)\left(3+\cos 4 \varphi_{0}\right) \cos \theta_{0} \sin ^{3} \theta_{0} \\
+\frac{1}{4} A_{2 x y} \varepsilon_{x x}(t) \cos 2 \varphi_{0} \sin 2 \theta_{0} \\
\mu_{0} h_{2}(t)=A_{4 \varepsilon} \Delta \varepsilon(t) \sin 4 \varphi_{0} \sin ^{3} \theta_{0} \\
-\frac{1}{2} A_{2 x y} \varepsilon_{x x}(t) \sin 2 \varphi_{0} \sin \theta_{0}
\end{gathered}
$$

When taking into account $A_{2 x y}$, the SAW-induced rf fields now depend on the angle of the magnetization $\varphi_{0}$, which very closely follows that of the applied field. A dependence of the acoustic attenuation variations is therefore expected when varying the angle of the field, as observed experimentally [Fig. 7(a)]. 
[1] S. Tehrani, J. Slaughter, M. Deherrera, B. Engel, N. Rizzo, J. Salter, M. Durlam, R. Dave, J. Janesky, B. Butcher, K. Smith, and G. Grynkewich, Proc. IEEE 91, 703 (2003).

[2] T. Devolder and C. Chappert, J. Appl. Phys. 95, 1933 (2004).

[3] C. Maunoury, T. Devolder, C. K. Lim, P. Crozat, C. Chappert, J. Wecker, and L. Bar, J. Appl. Phys. 97, 074503 (2005).

[4] C. Papusoi, B. Delaet, B. Rodmacq, D. Houssameddine, J.-P. Michel, U. Ebels, R. C. Sousa, L. Buda-Prejbeanu, and B. Dieny, Appl. Phys. Lett. 95, 072506 (2009).

[5] C. H. Back, D. Weller, J. Heidmann, D. Mauri, D. Guarisco, E. L. Garwin, and H. C. Siegmann, Phys. Rev. Lett. 81, 3251 (1998).

[6] J. Miltat, G. Abuquerque, and A. Thiaville, Spin Dynamics in Confined Magnetic Structures (Springer, Berlin, 2001).

[7] T. Gerrits, H. A. M. Van Den Berg, J. Hohlfeld, L. Bär, and T. Rasing, Nature (London) 418, 509 (2002).

[8] H. W. Schumacher, C. Chappert, P. Crozat, R. C. Sousa, P. P. Freitas, J. Miltat, J. Fassbender, and B. Hillebrands, Phys. Rev. Lett. 90, 017201 (2003).

[9] C. H. Back, Science 285, 864 (1999).

[10] M. Bauer, R. Lopusnik, J. Fassbender, and B. Hillebrands, Appl. Phys. Lett. 76, 2758 (2000).

[11] S. Kaka and S. E. Russek, Appl. Phys. Lett. 80, 2958 (2002).

[12] Y. Acremann, M. Buess, C. H. Back, M. Dumm, G. Bayreuther, and D. Pescia, Nature (London) 414, 51 (2001).

[13] T. Devolder, M. Belmeguenai, H. W. Schumacher, C. Chappert, and Y. Suzuki, MRS Proc. 746, Q8.4 (2002).

[14] A. Kent, B. Özyilmaz, and E. Del Barco, Appl. Phys. Lett. 84, 3897 (2004).

[15] S. Garzon, L. Ye, R. A. Webb, T. M. Crawford, M. Covington, and S. Kaka, Phys. Rev. B 78, 180401 (2008).

[16] T. Devolder, C. Chappert, J. A. Katine, M. J. Carey, and K. Ito, Phys. Rev. B 75, 064402 (2007).

[17] T. Dietl, H. Ohno, and F. Matsukura, Phys. Rev. B 63, 195205 (2001).

[18] M. Bombeck, A. S. Salasyuk, B. A. Glavin, A. V. Scherbakov, C. Brüggemann, D. R. Yakovlev, V. F. Sapega, X. Liu, J. K. Furdyna, A. V. Akimov, and M. Bayer, Phys. Rev. B 85, 195324 (2012).

[19] L. Thevenard, C. Gourdon, J. Y. Prieur, H. J. von Bardeleben, S. Vincent, L. Becerra, L. Largeau, and J.-Y. Duquesne, Phys. Rev. B 90, 094401 (2014).

[20] L. Thevenard, J.-Y. Duquesne, E. Peronne, H. J. von Bardeleben, H. Jaffrès, S. Ruttala, J.-M. George, A. Lemaître, and C. Gourdon, Phys. Rev. B 87, 144402 (2013).

[21] W. Li, B. Buford, A. Jander, and P. Dhagat, IEEE Trans. Magn. 50, 3100704 (2014).
[22] L. Thevenard, I. S. Camara, P. Rovillain, A. Lemaître, C. Gourdon, and J.-Y. Duquesne, Phys. Rev. B 93, 140405(R) (2016).

[23] R. Street and J. C. Woolley, Proc. Phys. Soc. London, Sect. A 62, 562 (1969).

[24] Given the pixel size and the setup magnification, 1 pixel corresponds to $0.26 \mu \mathrm{m}$ on the sample.

[25] D. Royer and E. Dieulesaint, Elastic Waves in Solids I: Free and Guided Propagation, Advanced Texts in Physics (Springer, Springer-Verlag, Berlin, Heidelberg, 2000).

[26] H. Bandömmel amd K. Dransfeld, Phys. Rev. Lett. 3, 83 (1959).

[27] M. Weiler, L. Dreher, C. Heeg, H. Huebl, R. Gross, M. Brandt, and S. T. B. Goennenwein, Phys. Rev. Lett. 106, 117601 (2011).

[28] W. K. Hiebert, L. Lagae, and J. De Boeck, Phys. Rev. B 68, 020402 (2003).

[29] B.-C. Choi, M. Belov, W. K. Hiebert, G. E. Ballentine, and M. R. Freeman, Phys. Rev. Lett. 86, 728 (2001).

[30] Calculated at $20 \mathrm{~K}$ assuming a Bloch domain wall of energy $\sigma=4 \sqrt{A_{\mathrm{ex}} K_{u}}$ with $A_{\mathrm{ex}}=8 \times 10^{-14} \mathrm{~J} \mathrm{~m}^{-1}$ from measurements done on similar samples [42], and $K_{u}=1228 \mathrm{~J} \mathrm{~m}^{-3}$ measured by cavity-FMR.

[31] Y. Shiota, T. Nozaki, F. Bonell, S. Murakami, T. Shinjo, and Y. Suzuki, Nat. Mater. 11, 39 (2011).

[32] We are comparing here SAW FMR data taken in repetitive mode with Kerr data taken in single-shot mode. Kerr data taken in repetitive mode showed that magnetization switching then starts at slightly lower fields than in single-shot mode.

[33] K. Gnatzig, H. Dotsch, M. Ye, and A. Brockmeyer, J. Appl. Phys. 62, 4839 (1987).

[34] R. D. McMichael and P. E. Wigen, J. Appl. Phys. 64, 5474 (1988).

[35] D. J. Seagle, S. H. Charap, and J. O. Artman, J. Appl. Phys. 57, 3706 (1985).

[36] J. Wunderlich, A. C. Irvine, J. Zemen, V. Holý, A. W. Rushforth, E. De Ranieri, U. Rana, K. Výborný, J. Sinova, C. T. Foxon, R. P. Campion, D. A. Williams, B. L. Gallagher, and T. Jungwirth, Phys. Rev. B 76, 054424 (2007).

[37] M. Birowska, C. Śliwa, J. A. Majewski, and T. Dietl, Phys. Rev. Lett. 108, 237203 (2012).

[38] H. Riahi, L. Thevenard, M. Maaref, B. Gallas, A. Lemaître, and C. Gourdon, J. Magn. Magn. Mater. 395, 340 (2015).

[39] A. J. Slobodnik et al., Electron. Lett. 8, 307 (1972).

[40] J. S. Blakemore, J. Appl. Phys. 53, R123 (1982).

[41] L. Dreher, M. Weiler, M. Pernpeintner, H. Huebl, R. Gross, M. S. Brandt, and S. T. B. Goennenwein, Phys. Rev. B 86, 134415 (2012).

[42] S. Shihab, H. Riahi, L. Thevenard, H. J. von Bardeleben, A. Lemaître, and C. Gourdon, Appl. Phys. Lett. 106, 142408 (2015). 